\title{
BMJ Open Mapping structure, process and \\ outcomes in the removal of low-value care practices in Canadian intensive care units: protocol for a mixed-methods exploratory study
}

\author{
Jeanna Parsons Leigh (D) ,, ${ }^{1,2}$ Jennie Petersen, ${ }^{2}$ Chloe de Grood, ${ }^{3}$ \\ Liam Whalen-Browne, ${ }^{2}$ Daniel Niven, ${ }^{2}$ Henry Thomas Stelfox ${ }^{2,3}$
}

To cite: Parsons Leigh J, Petersen J, de Grood C, et al. Mapping structure, process and outcomes in the removal of low-value care practices in Canadian intensive care units: protocol for a mixed-methods exploratory study. BMJ Open 2019;9:e033333. doi:10.1136/ bmjopen-2019-033333

- Prepublication history for this paper is available online. To view these files, please visit the journal online (http://dx.doi. org/10.1136/bmjopen-2019033333).

Received 01 August 2019 Revised 25 November 2019 Accepted 27 November 2019

Check for updates

(C) Author(s) (or their employer(s)) 2019. Re-use permitted under CC BY-NC. No commercial re-use. See rights and permissions. Published by BMJ.

${ }^{1}$ Department of Medicine, Schulich School of Medicine and Dentistry, Western University, London, Ontario, Canada ${ }^{2}$ Department of Critical Care Medicine, University of Calgary Cumming School of Medicine, Calgary, Alberta, Canada ${ }^{3}$ Department of Community Health Sciences, University of Calgary Cumming School of Medicine, Calgary, Alberta, Canada

Correspondence to Dr Jeanna Parsons Leigh; Jeanna.ParsonsLeigh@uwo.ca

\section{ABSTRACT}

Introduction The challenge of implementing best evidence into clinical practice is a major problem in modern healthcare that can result in ineffective, inefficient and unsafe care. There is a growing body of literature which suggests that the removal or reduction of low-value care practices (ie, deadoption) is integral to the delivery of high-quality care and the sustainability of our healthcare system. However, currently very little is known about deadoption practices in Canada. We propose to map the current state of deadoption in Canadian intensive care units (ICUs). A key deliverable of this work will include development of an inventory of barriers, facilitators and potential implementation strategies for guiding the deadoption efforts.

Methods and analysis We will use Canadian adult general systems ICUs as our laboratory of investigation and employ a two-phased sequential exploratory mixedmethods approach: (1) semi-structured interviews with critical care stakeholders to develop an understanding of the structure (ie, healthcare context), process (ie, actions and events in healthcare) and outcomes (ie, effects on health status, quality, knowledge or behaviour) of deadoption (phase I) and (2) surveys with a broader sample of critical care stakeholders to further identify important barriers and facilitators, as well as potential implementation strategies (phase II). Interview data will be analysed through qualitative content analysis and survey data will be analysed through quantitative analyses to identify top barriers and facilitators, as well as top rated strategies.

Ethics and dissemination Ethical approval has been obtained through the University of Calgary Research Ethics Board (REB 17-2153). Participants involved will have the opportunity to provide feedback on the final written reports to support accurate representation of the data. The findings of this study will be disseminated through peer-reviewed publications and oral presentations with critical care stakeholders across Canada. Patient and family partners will receive an executive summary of the findings.

\section{Strengths and limitations of this study}

- The inclusion of decision-makers, physicians and nurses in our sample will help illuminate contextual factors influencing deadoption practices.

- Sampling in academic and non-academic hospital settings across five geographic regions in Canada will provide additional breadth in understanding facilitators and barriers to deadoption.

- The mixed-methods approach will serve to create a more cohesive and complete understanding of the deadoption process.

- Recruitment of interview and survey participants will be limited to those who can be reached through existing networks, thus the survey sample may not be a representative sample.

\section{INTRODUCTION}

\section{Background}

To provide the best healthcare to patients, the translation of best evidence into clinical practice is vital. This is especially true in the context of critical care medicine where the most advanced technology and treatments are often necessary to save lives. ${ }^{1-3}$ There is a significant delay between the development of scientific evidence and its implementation into clinical practice $\left(\sim 17\right.$ years) ${ }^{45}$ Delay in the translation of scientific information into clinical practice remains a challenge for healthcare systems as it creates gaps between best evidence available and the way clinical practices are delivered. ${ }^{6}$ Low-value care may be described include three categories of practice where the best available evidence suggests that there is (1) little to no benefit for patients (ineffective), (2) the benefits provided is not proportional to the cost (inefficient) or (3) the risk of harm exceeds the potential benefit (unsafe). ${ }^{78}$ 


\section{Deadopting low-value clinical practices}

Previous efforts in the area of implementation science that aim to close knowledge-care gaps have focused on adoption, or the implementation of new high-value practices into clinical practice. However, recent work in this area has emphasised the importance of deadopting (eg, reducing) the use of practices that are of low-value (no longer supported by best scientific evidence) from clinical practice. ${ }^{9-11}$ Two types of knowledge gaps in particular, overuse (use of an intervention despite strong evidence indicating it is ineffective or harmful) and misuse (use of an intervention that scientific evidence has demonstrated to be effective, but for the wrong patients or under the wrong circumstances) highlight the need for deadoption. Both of these types of gaps indicate instances where an evidence supported intervention is used incorrectly, for the wrong patient or under the wrong circumstances and have critical implications on patient healthcare outcomes. ${ }^{12}$ For example, Pearson and Littlejohns showed that in the USA, patients in acute care receive recommended care only $53 \%$ of the time, leading to poor management of health conditions and preventable patient death. ${ }^{13}$ Meanwhile, numerous high-quality studies have demonstrated no meaningful effect using albumin during fluid resuscitation for most critically ill patients. ${ }^{14} 15$ Despite this, albumin continues to be prescribed to a high number of patients who need fluid resuscitation. ${ }^{16}$

\section{A focus on critical care medicine}

Intensive care units (ICUs) look after the most vulnerable patients in the hospital where rapidly changing technologies are needed to provide treatment to prevent further morbidity and mortality. Adult ICU admissions have increased by $12 \%$ in the last 10 years. ${ }^{17}$ In Canada, ICU costs are estimated to be $\sim \mathrm{CAD} \$ 6$ billion a year. ${ }^{17}$ The average daily cost of an ICU stay has been reported to be three times that of a stay in a general hospital ward. ${ }^{18}$ Due to both the ageing population in Canada and increased severity of patient illness, it is expected that the use and cost of critical care in Canada will continue to increase. ${ }^{10}$ Recent studies also indicate that ICUs already frequently operate close to capacity levels, limiting possibilities for units to accommodate for rising admission rates. ${ }^{19-21}$ Further, patients in the ICU are often frail with little ability to tolerate ineffective or harmful practices. ${ }^{2} 20$ Research to build a foundation to guide the deadoption of low-value practices in critical care medicine is both timely and necessary given that ICUs currently use the highest number of expensive therapies, technologies and treatments in the health system in an effort to save lives. ${ }^{19}$ Reduction of costs to the healthcare system through removing practices no longer supported by best evidence in critical care medicine is necessary to reduce low-value care.

\section{Deadoption of low-value clinical practices in critical care medicine}

Campaigns such as Choosing Wisely-which has prioritised the identification of low-value care practices in critical care medicine $^{21}$ - have been highly publicised ${ }^{22}$ and have influenced healthcare priorities at provincial, national and international levels. ${ }^{23}{ }^{24}$ However, little is known about how best to facilitate and sustain the deadoption of these practices. ${ }^{22}$ Purely technical approaches have been shown to be insufficient, as they often neglect the impact of social, ethical, cultural and preferential factors. ${ }^{25}$ Given the recent Choosing Wisely campaign and raised attention level to low-value care in critical care medicine,${ }^{26}$ this programme of work is timely, since a lack of knowledge of how to apply and execute principles of deadoption in a critical care context risks efforts being ineffective (eg, a deadoption strategy that decays over time because it is not tailored to the local setting), or having unintended consequences (eg, a deadopted practice replaced by another low-value practice). To address this gap in implementation science, we propose a programme of research that uses Canadian ICUs as a laboratory to develop an evidence base by mapping the structure (ie, healthcare context), process (ie, actions and events in healthcare) and outcomes (ie, effects on health status, quality, knowledge or behaviour ${ }^{27}$ of current approaches to deadoption in Canadian critical care medicine.

\section{Overall objective}

To understand factors associated with the deadoption of low-value clinical practices in critical care medicine and develop an evidence base of facilitators, barriers and potential implementation strategies to guide future deadoption efforts. To achieve our objective, we will use Canadian adult ICUs as our laboratory of investigation and apply the Donabedian model of healthcare quality. ${ }^{27}$ Donabedian's model has previously been validated in other acute care contexts (ie, trauma) where improvements in care structures and processes were associated with beneficial effects on patient outcomes and use of resources. ${ }^{28}$ In this study, we will employ a two-phased sequential exploratory mixed-methods approach: (1) one-to-one interviews with critical care stakeholders to understand the current structure (ie, context), process (ie, interactions between stakeholders) and outcomes (ie, impact of deadoption efforts) ${ }^{27}$ in ICUs across Canada (phase I) and (2) surveys with the broader critical care community to further identify additional barriers, facilitators and potential implementation strategies not published in the literature (phase II). This work also builds on an in-progress systematic review to identify published barriers and facilitators to deadoption in acute care (registered in PROSPERO: http://www.crd.york.ac.uk/PROSPERO/display_ record.php? ID=CRD42016050234). Findings from both phases of research will be integrated with the results of the systematic review and synthesised into an inventory of barriers, facilitators and targeted implementation strategies to map the current state of deadoption in Canadian critical care medicine. The protocol for this manuscript is informed by initial findings from the systematic review also involving members of this team (Parsons-Leigh, Niven and Stelfox). Phase I of this work is intended to 


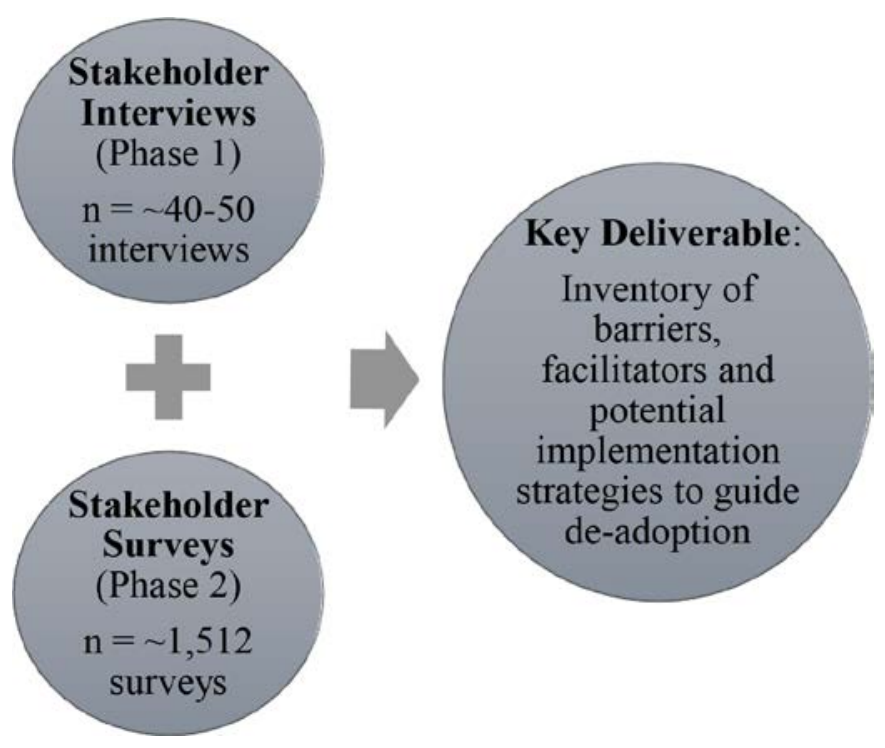

Figure 1 Overview of study protocol and key deliverables.

start in October of 2019, followed by phase II in June 2020.

\section{Specific aims}

\section{Aim 1}

To describe current deadoption practices in Canadian adult ICUs and identify barriers and facilitators related to deadoption from the perspective of ICU stakeholders (phases I and II).

\section{Aim 2}

To develop an inventory of barriers, facilitators and potential implementation strategies to guide future deadoption efforts in critical care medicine (phases I-II and deliverable).

\section{METHODS AND ANALYSIS}

\section{Overview of planned research in Canadian ICUs}

The primary aim of this programme of work is to build an evidence base to guide the process of deadoption in critical care medicine on a national scale. A map of the structure, processes and outcomes of deadoption in critical care settings will be developed based on the findings from two phases of research using a sequential exploratory methodology, which involves interviews (phase I) and surveys (phase II) with stakeholders working in critical care settings across Canada (figure 1). We will also utilise the findings from the published literature on deadoption, ${ }^{29}$ including the systematic review (see Overall Objective) currently taking place, to identify barriers, facilitators and potential implementation strategies to support the deadoption of low-value clinical practices. Canadian adult general system ICUs (ie, ICUs that provide care to a mix of illness types but are primarily medical and surgical patients) will be used as our laboratory of investigation to evaluate and refine the barriers, facilitators and implementation strategies within a clinical context. The findings from this research will help capture the complexity of the deadoption process and will ultimately support clinical and health policy decision-making in critical care medicine. We define barriers and facilitators as factors that influence the discontinuation of a clinical practice (ie, healthcare delivery acts such as diagnosis, therapeutic interventions, healthcare technology, education and prevention efforts) after it has been previously adopted. ${ }^{11}$ Furthermore, we divide low-value clinical practices into three categories: ineffective (ie, do not produce any significant or desired effect), inefficient (ie, where the cost is not proportional to the benefits) or harmful (ie, where the risk of harm outweighs any benefit). ${ }^{78} 29$ We define ICU as a distinct hospital care unit staffed by specialised professionals where immediate and continuous life-sustaining treatment is provided to patients. This protocol is the foundational step in a programme of research that will launch a systematic, customisable and sustainable deadoption strategy for ICUs across Canada.

\section{Patient and public involvement}

Patient and family partners already affiliated with our group were involved in the design of this study, participated as coinvestigators on the subsequently funded grant application, and will meet with the research team to discuss emerging themes during the data collection phase. The results of this study will be disseminated to all study collaborators through a written executive summary and potentially oral presentations if there is interest. Members of the general public were not involved in the design of this study.

\section{Phase I: stakeholder interviews}

\section{Objective}

To characterise deadoption practices in Canadian general system ICUs, including the structure, process and outcomes for the removal of low-value care practices.

\section{Design}

Individual interviews will be conducted with a purposive sample of ICU administrators (unit managers, medical directors) and frontline providers (physicians, nurses) from at least two hospitals in five Canadian provinces ( $\mathrm{n}=10$ hospitals) with general systems ICUs to further explore their experiences of deadoption. The interview question guide will be informed by the barriers, facilitators and potential implementation strategies identified in the published literature, as well as in the systematic review (discussed earlier) in an effort to further understand the relevance of top-rated factors in the context of the current state of deadoption decision-making. Furthermore, the interview guide will be structured based on the Donabedian model and will include questions focused on exploring factors related to structure (ie, healthcare context), process (ie, actions and events in healthcare) and outcomes (ie, effects on health status, quality, knowledge or behaviour $)^{27}$ in the removal of low-value care practices. For example, we will ask stakeholders to describe the type of facility or institution (eg, teaching 
hospital vs community hospital; quality improvement staff support integrated into structure) they work in (structure) and how they perceive this to impact stakeholder actions or responses related to deadoption initiatives (process) and the effects of this on the use of low-value care (outcomes). The interview guide will be piloted and iteratively refined with four to five local critical care medicine stakeholders in the Calgary prior to conducting interviews with stakeholders from additional hospitals and provinces in Canada.

\section{Sampling and recruitment}

Participants will be purposively sampled to ensure representation of both urban and regional ICUs and academic and non-academic ICUs from various Canadian geographic regions to maximise the diversity of perspectives and experiences captured. We will target five provinces (Alberta, AB; British Columbia, BC; Nova Scotia, NS; Ontario, ON; Quebec, QC) that represent 4/5 of Canada's major geographic regions: West (BC), Prairie (AB), Central (ON, QC) and Atlantic (NS). We will start with our team's home province of Alberta and to initially ensure our design will capture a diverse range of perspectives, we will recruit at least four ICUs across the province ( $\mathrm{n}=16-24$ in Alberta). Our team has previously compiled a comprehensive list of Canadian adult ICU medical directors and unit managers that was successfully used to survey administrators in a study of ICU rounding practices. ${ }^{30}$ This list will be reviewed for accuracy, updated based on any publically available information and used to identify potential interviewees. We will use snowball sampling based on this list to request participation of 4-5 participants from each ICU, including 1 medical director or unit manager, 2 physicians and 1-2 nurses from 1 academic and 1 non-academic hospital in the 5 Canadian provinces (except in Alberta, we will recruit at least 2 academic and 2 non-academic hospitals) to participate in the interview process (4-5 participants $\times 2$ hospitals $\times 4$ provinces $+4-5$ participants $\times 4$ hospitals in Alberta $=48-60$ participants in total). Contact will be initially made through telephone calls to either the medical director or unit manager to request their participation and to ask for their help in forwarding the study information to physicians and nurses in their ICU. Participants who consent to the study will be contacted for an interview. If we are not able to initially achieve our target sample as described above, we will follow-up with the medical director or unit manager to ask for their help in obtaining the proposed sample frame in their ICU. Interview guides and transcripts will be translated into French as necessary by a skilled translator and then checked for accuracy by a bilingual healthcare professional. Non-respondents will be contacted up to three times at 2-week intervals to set up an interview. If selected participants do not respond within this timeframe, we will contact the next person on our list of consenting participants with like characteristics. Interviews will last $\sim 30-45 \mathrm{~min}$.

\section{Study sample}

We will target a total of 12 ICUs (2 per province $\times 4+4$ in Alberta=12), including completion of $\sim 12$ interviews with ICU administrators $(\mathrm{n}=12$ medical directors/unit managers) and $36-48$ frontline providers $(n=18-24$ physicians and $n=18-24$ nurses), for a total of 48-60 interviews completed (4-5 interviews for 2 hospital settings in each of 4 provinces plus 2 additional hospitals in Alberta).

\section{Procedure}

Semi-structured individual telephone interviews with ICU administrators and frontline providers (physicians and nurses) will be conducted. Participants will be asked about the characteristics of deadoption decision-making in their ICU (eg, who is involved, how are stakeholders engaged, what did this look like, what are the drivers of non-involvement of patients or families, what are the strategies for improvement, what are the potential barriers to realising these strategies, how ICU structural elements influence deadoption processes and so on). Moreover, participants will be asked how low-value practices are selected of deadoption, what types of deadoption practices or initiatives are currently underway, successes and failures related to the deadoption processes, barriers and facilitators to deadoption, and effective strategies that have been used in their unit to successfully deadopt an ineffective or harmful clinical practice (eg, how are interventions developed, implemented, evaluated and sustained?).

\section{Data analysis}

Qualitative content analysis ${ }^{31}$ will be performed to describe participant perspectives of deadoption. All interviews will be audio taped, transcribed verbatim, assigned a unique identifier and imported into NVivo V.10. Two qualitative investigators will use a process of induction to transform data from individual sources to common, interactive themes involving coding, categorising and conceptualising. ${ }^{3233}$ Coders will independently review a sampling of the texts, and begin to formulate provisional themes and codes. Weekly coding meetings will facilitate a negotiated and refined coding framework for constant comparative analysis. ${ }^{34}$ A reference document defining each node of the coding framework will be created and modified to reflect coding discussions. Coders will examine and assign sections of text to codes, representing themes, and will identify discrepant and negative information. Written notes and memos will be taken throughout to help document the process and account for the decisions made during data analysis. The result will be a 'story' of deadoption that describes the structure, processes and outcomes of deadoption in critical care medicine, including the barriers and facilitators to deadoption, and opportunities for improvement. A copy of the study findings will be provided to interview participants for review/comment as a form of member checking. ${ }^{35}$ We plan to analyse $48-60$ telephone interviews but are prepared to collect additional data if thematic saturation is not achieved. ${ }^{35}$ 


\section{Deliverable}

A comprehensive description of stakeholder perceptions, and important contextual and institutional factors associated with deadoption in Canadian medical surgical ICUs.

\section{Phase II: stakeholder surveys \\ Objective}

To further describe the current state of deadoption practices in Canadian adult general system ICUs, including top barriers and facilitators, as well as potential implementation strategies to initiate, execute and sustain the removal of low-value practices.

\section{Design}

Individual stakeholder surveys will be administered to all ICU administrators (unit managers and medical directors) and a representative sample of frontline providers (physicians and nurses) across Canada to solicit their perceptions of and experiences with deadoption. Survey items will be generated from the barriers, facilitators and implementation strategies identified in both the systematic review (discussed earlier) and phase I, extending the generalisability of those findings. We will follow a systematic approach to survey development ${ }^{36}$ based on the Theoretical Domains Framework ${ }^{37}$ that includes an iterative process of feedback and refinement to tailor findings from both the published literature and phase I findings to the ICU environment. Survey instruments will be pilot tested with local ICU experts prior to administration.

\section{Sampling and recruitment}

The medical director or unit manager in each Canadian general system ICU $(n=180)$ will be emailed and asked to complete an electronic survey using secure online survey software (Survey Monkey, Palo Alto, California, USA). We will use a snowball recruitment strategy to recruit medical directors, unit managers, physicians and nurses in Canadian ICUs. Through initial contact with each medical director or unit manager, we will ask for their participation in the survey, as well as for their help in forward the survey to one additional administrator and $\sim 10$ providers from their ICU ( 5 physicians and 5 nurses). Our goal is to capture a diverse representation of those in leadership positions (unit manager, medical director), as well as frontline nurses (more experienced, less experienced) and attending physicians (male, female, senior level, junior level). Non-respondents will be sent up to three reminders at 2-week intervals.

\section{Study sample}

We will target distribution of $\sim 360$ surveys to ICU administrators $(n=180$ medical directors and $n=180$ unit managers) and 1800 surveys to frontline providers ( $n=900$ physicians and $n=900$ nurses ), for a total of 2160 surveys distributed (12 surveys per ICU for each of the 180 hospitals).

\section{Procedure}

Participants will be asked to rate the importance of barriers and facilitators that contribute to improving the deadoption of low-value care practices within their ICU, using a 7-point Likert scale. Through open-ended questions, survey respondents will also be asked for their ideas and thoughts on contextual factors that influence deadoption. The perceived effectiveness of potential strategies to initiate and sustain changes in practice within the ICU will be assessed by participants using a similar scale. Specific examples of both ineffective (eg, albumin for fluid resuscitation $)^{14}$ and harmful (eg, tight glycemic control $)^{11}$ clinical practices relevant to ICU care will be used throughout the survey to illustrate meaning. The top five barriers and top five facilitators, as well as the top five harmful or ineffective practices and top five implementation strategies appropriate for the deadoption of low-value practices will be identified by each participant through this survey. Finally, demographic data will be requested (ie, age, gender, number of years working in critical care, role in organisation, primary specialisation, type of institution) to ascertain if there are differences in perception related to provider characteristics (eg, medical director vs unit manager, physicians vs nurses) and hospital setting (ie, urban vs rural, academic vs non-academic).

\section{Data analysis}

A comprehensive list of barriers (eg, ICU resistance to change), facilitators (eg, local champion) and proposed implementation strategies (eg, onsite education) to deadoption reported in the individual surveys will be compiled through quantitative analysis. The importance of each barrier, facilitator and proposed implementation strategies will be illustrated by using medians with IQRs and proportions with binomial 95\% CIs. Detailed tabulations to compare characteristics by decision-maker (unit manager, medical director), provider (nurses, physicians) and hospital (urban vs rural, academic vs non-academic) will also be conducted. $\chi^{2}$ and KruskalWallis tests for institution comparisons and mixed-effects logistic regressions will be used to evaluate the significance of observed differences in reported barriers and facilitators for provider comparisons (data clustered by unit), although these comparisons are not the primary objective of this phase and likely to be underpowered. An earlier study that also surveyed stakeholders in the ICU identified a response rate of over $60 \%,{ }^{38}$ however, we have used a more conservative estimate of $40 \%$ to assume that 864 surveys (2160 surveys distributed with $40 \%$ target response rate) will be returned and used for quantitative analysis. This will provide a binomial CI of $\pm 4.6 \%$ (based on 864 surveys equally clustered across 180 ICUs) for global point estimates.

\section{Deliverable}

Clear understanding of the relative importance of individual barriers, facilitators and potential implementation 
strategies for the deadoption of low-value practices to Canadian ICU decision-makers and frontline providers.

\section{Mapping structure, process and outcomes in deadoption}

Following stakeholder interviews, data from both phases of work will be synthesised to map the process, structure and outcomes of deadoption efforts in Canadian medical surgical ICUs. Specifically, the interview and survey findings will be synthesised into an inventory of key factors, barriers, facilitators and proposed implementation strategies to guide the deadoption of both ineffective (eg, albumin for fluid resuscitation) and harmful (eg, tight glycemic control) clinical practices. Findings from the systematic review (mentioned earlier) will also be integrated into this final deliverable. Barriers, facilitators and proposed implementation strategies pertaining to deadoption will also be characterised based on their appropriateness to different practice settings (academic vs non-academic), providers (physicians vs nurses) and decision-maker (medical director vs unit manager) characteristics. Interview participants will be given an opportunity to provide feedback on the draft findings as a form of member checking. To date, contextual and institutional factors that influence the removal of low-value clinical practices from patient care are simply not known. The results of this work will provide researchers, providers, and clinical and health policy decision-makers with vital information to launch informed, evidence-based deadoption initiatives. Moreover, while this research will be conducted in the context of Canadian adult ICUs, we believe it will be transferable to other acute care contexts and multidisciplinary healthcare settings, although this will need to be confirmed in future studies.

Mapping the structure, process and outcomes of deadoption is a first step towards eliminating low-value practices from patient care as a means of fostering the delivery of safe, effective and sustainable care. We are committed to testing and implementing our deadoption strategy through a series of future projects:

1. Prospectively validate the results of this work in a new sample of ICU stakeholders. We intend to seek funding for this next phase of work during the third year of the proposed study.

2. Test the map of deadoption structures, processes and outcomes (pilot trial followed by multicentre cluster randomised controlled trial) by using it to develop and implement a multifaceted implementation strategy to deadopt a single low-value clinical practice in Canadian general system ICUs.

3. Evaluate the findings of this work in other clinical and acute care contexts (eg, surgery) ${ }^{39}$ and adapt as required.

\section{ETHICS AND DISSEMINATION}

\section{Ethical considerations}

Informed consent will be obtained from all critical care stakeholders involved in the interviews prior to any data collection. All interview participants will receive a $\$ 25$ gift card as a form of compensation for their time investment in the study. Completion of the survey will imply consent. All personal identifiers will be removed in any publication or presentation of the data to protect the confidentiality of the participants and ICU locations. Participants will also have the opportunity to review their deidentified interview transcripts and provide feedback on the results, including the draft framework before it is finalised to ensure accurate representation of their perspectives, experiences and voices.

\section{Dissemination plans}

Drawing on the Ottawa Model for Research Use, ${ }^{40}$ all members of the research team, including knowledge users, will play an integral role throughout the entire research programme. Our investigative team's alignment with a diverse group of stakeholders will remove barriers between academia and the healthcare system, facilitating collaboration and advancements in implementation science for system enhancement. Healthcare providers and decision-makers will be active stakeholders and will be engaged at all times through this work, from planning to end of project. We will disseminate an executive summary of the research, along with the framework at the completion of this study to all stakeholders involved. A patient representative has supported the design of this study, including development of the methods and approach and will be engaged in the knowledge dissemination planning process for patient and families. We plan to share results with patient and family partners in the form of written executive summaries and potentially oral presentation if there is interest. Results of the study will also be shared with the Canadian Critical Care Society (https://canadiancriticalcare.org), the Canadian Critical Care Trials Group (https://www.ccctg.ca/) and more broadly to informal networks in the community through traditional peer-review publications and oral presentations at upcoming meetings and conferences.

\section{CONCLUSION}

Ineffective, inefficient or potentially harmful practices are common in healthcare due in part to the complexity and difficulty associated with the translation of scientific evidence into clinical practice. ${ }^{11}$ This programme of work will map prevailing opinions pertaining to deadoption of low-value clinical practices in adult ICUs in Canada, determine barriers and facilitators to deadoption in general system ICUs and provide hospitals with an applied framework to guide the deadoption process. Canadians who are critically ill are the sickest and most vulnerable patients in the healthcare system, and thus, it is imperative they receive the right care at the right time. This project will help to build a foundation for the creation of effective strategies to improve the use of best evidence in critical care by facilitating the removal of practices that are no longer supported by scientific study. 
Contributors All authors contributed to the conception (JPL, DN, HTS) or design (JPL, JP, CdG, LW-B, HTS) of the work; and drafting (JPL, JP, CdG) or revising (DN, LWB, HTS) the work for important intellectual content; and provided final approval of the manuscript and agree to be accountable for the accuracy and integrity of the work. *Note: the initials above correspond to the authors of this manuscript as follows: JPL, JP, CdG, LW-B, DN, HTS

Funding This work was supported by Canadian Institutes of Health Research (CIHR Grant \#: 314548).

Competing interests None declared.

Patient consent for publication Not required

Ethics approval Research ethics board approval has been obtained from the Conjoint Health Research Ethics board (CHREB), University of Calgary (REB17-2153).

Provenance and peer review Not commissioned; externally peer reviewed.

Open access This is an open access article distributed in accordance with the Creative Commons Attribution Non Commercial (CC BY-NC 4.0) license, which permits others to distribute, remix, adapt, build upon this work non-commercially, and license their derivative works on different terms, provided the original work is properly cited, appropriate credit is given, any changes made indicated, and the use is non-commercial. See: http://creativecommons.org/licenses/by-nc/4.0/.

\section{ORCID iD}

Jeanna Parsons Leigh http://orcid.org/0000-0002-8408-674X

\section{REFERENCES}

1 Wennberg JE. Unwarranted variations in healthcare delivery: implications for academic medical centres. BMJ 2002;325:961-4.

2 Halpern NA, Pastores SM. Critical care medicine in the United States 2000-2005: an analysis of bed numbers, occupancy rates, payer mix, and costs*. Crit Care Med 2010;38:65-71.

3 Fowler RA, Abdelmalik P, Wood G, et al. Critical care capacity in Canada: results of a national cross-sectional study. Crit Care $2015 ; 19$.

4 Institute of Medicine Committee on the Quality of Health Care in America. Crossing the quality chasm: a new health system for the 21st century. Washington DC: National Academy Press, 2001.

5 Grant J. Evaluating "payback" on biomedical research from papers cited in clinical guidelines: applied bibliometric study. BMJ 2000;320:1107-11

6 Yokote G, Utterback R. Time lapses in information dissemination: research laboratory to physician's office. Bull Med Libr Assoc 1974;62:251-7.

7 Elshaug AG, Rosenthal MB, Lavis JN, et al. Levers for addressing medical underuse and overuse: achieving high-value health care. Lancet 2017;390:191-202.

8 Elshaug AG, Watt AM, Mundy L, et al. Over 150 potentially low-value health care practices: an Australian study. Med J Aust 2012;197:556-60.

9 Sibley JC, Sackett DL, Neufeld V, et al. A randomized trial of continuing medical education. N Engl J Med 1982;306:511-5.

10 Cabana MD, Rand CS, Powe NR, et al. Why don't physicians follow clinical practice guidelines? A framework for improvement. JAMA 1999;282:1458-65.

11 Niven DJ, Mrklas KJ, Holodinsky JK, et al. Towards understanding the de-adoption of low-value clinical practices: a scoping review. BMC Med 2015;13:255.

12 Polisena J, Clifford T, Elshaug AG, et al. Case studies that illustrate disinvestment and resource allocation decision-making processes in health care: a systematic review. Int J Technol Assess Health Care 2013;29:174-84.

13 Pearson S, Littlejohns P. Reallocating resources: how should the National Institute for health and clinical excellence guide disinvestment efforts in the National health service? J Health Serv Res Policy 2007;12:160-5.
14 Finfer S, Bellomo R, Boyce N, et al. A comparison of albumin and saline for fluid resuscitation in the intensive care unit. $N$ Engl $\mathrm{J} \mathrm{Med}$ 2004;350:2247-56.

15 Caironi P, Tognoni G, Masson S, et al. Albumin replacement in patients with severe sepsis or septic shock. N Engl J Med 2014;370:1412-21.

16 Jones D, Mcevoy S, Merz TM, et al. International albumin use: 1995 to 2006. Anaesth Intensive Care 2010;38:266-73.

17 Canadian Institutes of Health Research. About IHSPR. Available: http://www.cihrirsc.gc.ca/e/13948.html [Accessed 3 Oct 2018].

18 Elshaug AG, Moss JR, Littlejohns P, et al. Identifying existing health care services that do not provide value for money. Med J Aust 2009;190:269-73.

19 Niven DJ, McCormick J, Straus SE, et al. Reversal of scientific evidence and identifying opportunities for de-adoption in critical care: a scoping review. Am J Respir Med 2016;193.

20 Canadian Institutes for Health Information. Care in Canadian ICU's. CIHI: Ottawa ON, 2016.

21 Choosing Wisely. Critical Care Societies Collaborative - Critical Care 2016. Available: http://www.choosingwisely.org/societies/criticalcare-societies-collaborative-critical-care/ [Accessed 3 Oct 2018]

22 Elshaug AG, McWilliams JM, Landon BE. The value of low-value Lists. JAMA 2013;309:775-6.

23 Royal College of Physicians and Surgeons of Canada. Think again: choosing wisely campaign takes root in Canada, 2014. Available: http://www.royalcollege.ca/portal/page/portal/rc/resources/ publications/dialogue/vol14_4/ch oosing_wisely [Accessed 3 Oct 2018].

24 Morden NE, Colla CH, Sequist TD, et al. Choosing wisely - the politics and economics of labeling low-value services. N Engl J Med 2014;370:589-92.

25 Elshaug AG, Watt AM, Moss JR, et al. Policy perspectives on the obsolescence of health technologies in Canada: discussion paper October 2009, 2009. Available: https://www.cadth.ca/media/pdf/ Obsolescence of Health Technologies in Canada_Policy_Forum_e. pdf [Accessed 3 Oct 2018].

26 Kleinpell R, Sessler CN, Wiencek C, et al. Choosing wisely in critical care: results of a national survey from the critical care societies collaborative. Crit Care Med 2019;47:331-6.

27 Donabedian A. Evaluating the quality of medical care. Milbank Quarterly 2005;83:691-729.

28 Moore L, Lavoie A, Bourgeois G, et al. Donabedian's structureprocess-outcome quality of care model: validation in an integrated trauma system. J Trauma Acute Care Surg 2015;78:1168-75.

29 Parsons Leigh J, Niven DJ, Boyd JM, et al. Developing a framework to guide the de-adoption of low-value clinical practices in acute care medicine: a study protocol. BMC Health Serv Res 2017;17:54.

30 Holodinsky JK, Hebert MA, Zygun DA, et al. A survey of rounding practices in Canadian adult intensive care units. PLoS One 2015;10:e0145408.

31 Bengtsson M. How to plan and perform a qualitative study using content analysis. NursingPlus Open 2016;2:8-14.

32 Glaser B, Strauss A. The discovery of grounded theory. Chicago: Aldine Publishing Company, 1967.

33 Lincoln YS, Guba EG, Pilotta JJ. Naturalistic inquiry. Beverly Hills, CA: Sage Publications, 1985: 438-9.

34 Boeije H. A purposeful approach to the constant comparative method in the analysis of qualitative interviews. Qual Quant 2002;36:391-409.

35 Green J, Thorogood N. Qualitative methods for health research. 2nd ed. Thousand Oaks, CA: Sage, 2009.

36 Kelley Ket al. Good practice in the conduct and reporting of survey research. Int J Qual Health Care 2003;15:261-6.

37 Atkins L, Francis J, Islam R, et al. A guide to using the theoretical domains framework of behaviour change to investigate implementation problems. Implementation Sci 2017;12:1-18.

38 Stelfox HT, Niven DJ, Clement FM, et al. Stakeholder engagement to identify priorities for improving the quality and value of critical care. PLoS One 2015;10:e0140141-13.

39 Hirshon JM, Risko N, Calvello EJB, et al. Health systems and services: the role of acute care. Bull World Health Organ 2013:91:386-8.

40 Graham ID, Logan J. Innovations in knowledge transfer and continuity of care. Can J Nurs Res 2004;36:89-103. 